\section{Time-Inconsistent Problems}

\section{Jiongmin Yong}

Consider the following initial value problem of an ordinary differential equation:

$$
\left\{\begin{array}{l}
\dot{X}(s)=f(s, X(s), u(s)), \quad s \in[t, T], \\
X(t)=x .
\end{array}\right.
$$

In this differential equation, $u(\cdot)$, the control, is selected from some class $\mathcal{U}[t, T]$ of measurable functions with values in a metric space $U$, and the solution $X(\cdot)$ of $(1)$ is called the state process. In applications, $X(s)$ could be the total wealth from some stocks, tonnes of fish in a large lake, or the location of a moving object at time $s$, and $u(\cdot)$ could be a trading strategy, a harvest rate, or a driving force. Different selections of controls $u(\cdot)$ lead to different state processes $X(\cdot)$. To measure the performance of the control toward a goal, one may introduce the following cost functional:

$$
J(t, x ; u(\cdot))=\int_{t}^{T} e^{-\delta(s-t)} g(X(s), u(s)) d s .
$$

In this integral, $g$ is the running cost rate and $e^{-\delta(s-t)}$ is the exponential discounting (with discount rate $\delta>0$ ). To find a control that minimizes the cost functional, a classical optimal control problem can be stated as follows.

Problem (C). For a given initial pair $(t, x) \in[0, T] \times \mathbb{R}^{n}$, find $\bar{u}(\cdot) \in \mathcal{U}[t, T]$ such that

$$
J(t, x ; \bar{u}(\cdot))=\inf _{u(\cdot) \in \mathcal{U}[t, T]} J(t, x ; u(\cdot)) \equiv V(t, x) .
$$

In the above, $\bar{u}(\cdot)$ is an optimal control and $V(\cdot, \cdot)$ is defined as the value function. Bellman's principle of optimality can be stated as: for any $(t, x) \in[0, T) \times \mathbb{R}^{n}$ and $\tau \in(t, T]$,

$$
\begin{aligned}
V(t, x)=\inf _{u(\cdot) \in \mathcal{U}[t, \tau]}\left\{\int_{t}^{\tau} e^{-\delta(s-t)}\right. & g(X(s), u(s)) d s \\
& \left.+e^{-\delta(\tau-t)} V(\tau, X(\tau))\right\} .
\end{aligned}
$$

This principle gives: if $\bar{u}(\cdot)$ is an optimal control at $(t, x)$ with $\bar{X}(\cdot)$ being the corresponding optimal state process,

Jiongmin Yong is a professor of mathematics at the University of Central Florida. His email address is jiongmin. yong@ucf. edu.

For permission to reprint this article, please contact:

reprint-permission@ams.org.

DOI: https://doi.org/10.1090/noti2242 then for any $\tau \in(t, T]$,

$$
\begin{aligned}
& V(t, x)=J(t, x ; \bar{u}(\cdot)) \\
& =\int_{t}^{\tau} e^{-\delta(s-t)} g(\bar{X}(s), \bar{u}(s)) d s+e^{-\delta(\tau-t)} J(\tau, \bar{X}(\tau) ; \bar{u}(\cdot)) \\
& \geqslant \int_{t}^{\tau} e^{-\delta(s-t)} g(\bar{X}(s), \bar{u}(s)) d s+e^{-\delta(\tau-t)} V(\tau, \bar{X}(\tau)) \\
& \geqslant V(t, x) .
\end{aligned}
$$

This implies

$$
V(\tau, \bar{X}(\tau))=J\left(\tau, \bar{X}(\tau) ;\left.\bar{u}(\cdot)\right|_{[\tau, T]}\right),
$$

which means the restriction $\left.\bar{u}(\cdot)\right|_{[\tau, T]}$ of an optimal control $\bar{u}(\cdot)$ selected for the initial pair $(t, x)$ on $[t, T]$ is an optimal control for the initial pair $(\tau, \bar{X}(\tau))$ on $[\tau, T]$. In other words, an optimal control $\bar{u}(\cdot)$ determined at time $t$ will stay optimal thereafter. Such a phenomenon is called the time-consistency of the control problem or the optimal control.

People frequently regret the decision they made earlier, meaning an optimal decision made today will hardly seem optimal forever. We call such a phenomenon the timeinconsistency of the problem under consideration. There are two major reasons causing time-inconsistency: timepreferences and risk-preferences. To elaborate a bit more, normal people usually are not $100 \%$ rational and they often over-weigh the immediate satisfaction level (the utility) or regard the immediate time period more precious. Here is an example: if you are invited to referee a paper, would you review the paper immediately or would you wait until the associate editor sends you a reminder? Time-preferences play a role here. Different people could have different opinions on upcoming uncertain events. An easy example to illustrate risk-preferences is the opinion on whether to buy a risky stock.

Mathematically, time-preferences can be described by discounting, and risk-preferences can be described by subjective probability. It is possible to present such problems in a stochastic setting. But for simplicity, let us continue with the deterministic case. Problem (C) (with exponential discounting) is time-consistent, which represents a situation in which the controller (the person who is controlling the system) is rational. Now if the controller is not $100 \%$ rational, then the discounting might not be exponential. A typical nonexponential discounting is hyperbolic discounting, which is illustrated as

$$
J(t, x ; u(\cdot))=\int_{t}^{T} \frac{1}{1+a(s-t)} g(X(s), u(s)) d s
$$

for some $a>0$. With such a cost functional, the corresponding optimal control problem will be timeinconsistent, i.e., if $\bar{u}(\cdot)$ is an optimal control for $(t, x)$ defined on $[t, T]$ with optimal state process $\bar{X}(\cdot)$, then there 
will be a later time $\tau \in(t, T]$ such that the optimal control $\hat{u}(\cdot)$ for $(\tau, \bar{X}(\tau))$ defined on $[\tau, T]$ does not satisfy the following:

$$
\hat{u}(s)=\bar{u}(s), \quad \text { a.e. } s \in[\tau, T] .
$$

For time-inconsistent optimal control problems, it is not wise to find optimal controls. Instead, one should look for equilibrium strategies. The idea is to regard the problem as a multiperson differential game in which today's self plays with future selves. To make the future reasonably satisfactory, today's self should be willing to sacrifice some immediate satisfaction. Saving for retirement is such an example. Under proper conditions, such equilibrium strategies can be constructed.

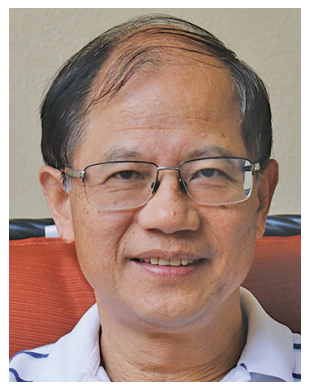

Jiongmin Yong

\section{Credits}

Photo of Jiongmin Yong is courtesy of Jiongmin Yong.

\section{FEATURED TITLES FROM HINDUSTAN
BOOK AGENCY}
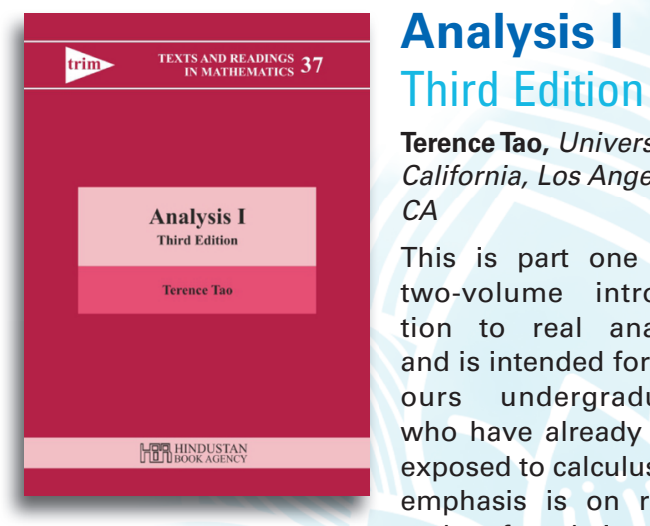

Terence Tao, University of California, Los Angeles, CA

This is part one of a two-volume introduction to real analysis and is intended for honours undergraduates who have already been exposed to calculus. The emphasis is on rigour and on foundations. The

material starts at the very beginning - the construction of the number systems and set theory-then goes on to the basics of analysis (limits, series, continuity, differentiation, Riemann integration), through to power series, several variable calculus and Fourier analysis, and finally to the Lebesgue integral.

Hindustan Book Agency; 2014; 368 pages;

Hardcover; ISBN: 978-93-80250-64-9; List US\$50;

AMS members US\$40; Order code HIN/66

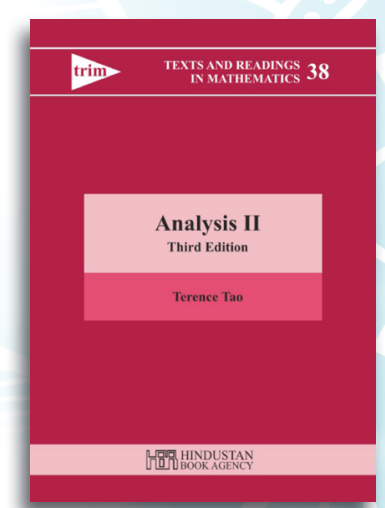

\section{Analysis II} Third Edition

Terence Tao, University of California, Los Angeles

This is part two of a two-volume introduction to real analysis and is intended for honours undergraduates who have already been exposed to calculus. The emphasis is on rigour and on foundations.

Hindustan Book Agency; 2014; 236 pages;

Hardcover; ISBN: 978-93-80250-65-6; List US\$40;

AMS members US\$32; Order code HIN/67

Titles published by the Hindustan Book Agency (New Delhi, India) include studies in advanced mathematics, monographs, lecture notes,

and/or conference proceedings on current topics of interest.

Discover more books at bookstore.ams.org/hin

Publications of Hindustan Book Agency are distributed within the Americas by the American Mathematical Society. Maximum discount of $20 \%$ for commercial channels.

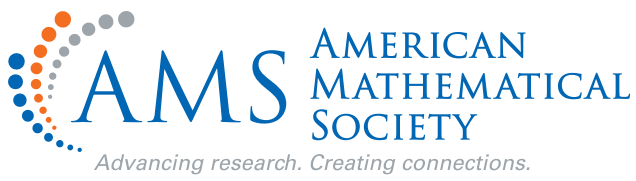

